\title{
Antioxidant Potential of Green and Black Teas of Selected South India Cultivars
}

\author{
Yuko TAKANO-ISHIKAWA ${ }^{1}$, Jun WATANABE ${ }^{1 *}$, Masao GOTO ${ }^{1}$, \\ Lingamallu Jagan Mohan RAO ${ }^{2}$ and Kulathooran RAMALAKSHMI ${ }^{2}$ \\ ${ }^{1}$ National Food Research Institute, National Agriculture and Food Research Organization (Tsukuba, \\ Ibaraki 305-8642, Japan) \\ ${ }^{2}$ Central Food Technological Research Institute (Mysore-570020, Karnataka, India)
}

\begin{abstract}
Tea is one of the most popular global beverages. It is widely consumed as a health promoting beverage and its health benefits are mainly due to the presence of polyphenols such as catechins, theaflavins and thearubigins. The purpose of the present study is to evaluate the antioxidant potential of green tea (GT) and black tea (BT) extracts of eight different cultivars by radical scavenging activity (DPPH-RSA) and hydrophilic oxygen radical absorbance capacity (H-ORAC). The extracts of teas were also analyzed for total polyphenol (TP) content and catechins. H-ORAC [ $(\mu \mathrm{mol}$ Trolox equivalent (TE)/g)] values ranged from 2730-5031 (GT), 1429-2766 (BT) and DPPH-RSA ( $\mu$ mol TE/g) ranged from 1098-1376 and 508-798, respectively. Comparing the same cultivar, GT showed higher antioxidant activities (H-ORAC and DPPH-RSA) than those of BT which is partially attributable to the increased TP and concentration of catechins. Correlation coefficients between parameters of antioxidant activities were TP/H-ORAC (GT: 0.647, BT: 0.79), TP/DPPH -RSA $(0.744,0.976)$ and DPPH-RSA/H-ORAC $(0.686,0.811)$ while those of BT exceeded those of GT. Extracts from all the cultivars showed good antioxidant potential. Antioxidant activities were mainly derived from catechins in GT and theaflavins in the case of BT.

Discipline: Food, Tea industry

Additional key words: Antioxidant activity, DPPH radical scavenging activity, polyphenols, ORAC
\end{abstract}

\section{Introduction}

Tea (Camellia sinensis var. assamica/sinensis), which belongs to the Theaceae family, is one of the most popular global beverages and widely consumed due to its health promoting characteristics, namely its anti-cancer, anti-bacterial, anti-viral and mainly antioxidant activity. Its health benefits are mainly due to the presence of polyphenols such as catechins, theaflavins and thearubigins. Fresh tea leaves contain catechins, including epicatechin, epigallocatechin, epicatechin gallate and epigallocatechin gallate.

During the preparation of green tea, fresh tea leaves are exposed to higher temperatures to deactivate/destroy the enzymes, resulting in green tea with high levels of catechins. Conversely, during the preparation of black tea, tea leaves have already undergone the oxidation process $^{21}$ otherwise known as fermentation. Polyphenol oxi- dase present in the green tea leaves oxidises catechins (flavanol monomers) to form flavanol polymers, (e.g. theaflavins and thearubigins) which leads to higher levels of theaflavins and thearubigins and lower levels of catechins present in black tea. Green and black teas also contain flavonols e.g. kaempferol, quercetin and myricetin ${ }^{5}$. The types and amounts of polyphenols present in tea differ depending on the variety of leaf, growing environment, processing, manufacturing, particle size of ground tea leaves and infusion preparation ${ }^{17,22}$.

The human body produces reactive oxygen species (ROS) by many enzymatic systems through oxygen consumption. Small amounts of ROS play a beneficial role as signal transducers ${ }^{16}$ and growth regulators ${ }^{4}$. However, excessive amounts of ROS oxidize numerous molecules, such as proteins, lipids and nucleotides and largely contribute to aging ${ }^{2}$, mutagenesis ${ }^{18}$ and coronary heart disease $^{3}$ through DNA breakage and oxidizing low-density lipoprotein ${ }^{24}$ which underlines the crucial need for exog-

*Corresponding author: e-mail nabej@affrc.go.jp

Received 29 November 2010; accepted 22 April 2011. 
enous antioxidants, such as vitamin $\mathrm{C}$, tocopherols and polyphenols?.

Tea leaves reportedly possess high antioxidant activities $^{13-15}$. However, few reports have compared the antioxidant activities and antioxidant compositions of different tea cultivars. In the present study, green and black teas from eight cultivars were collected from the same estate/plantation, having been grown under identical conditions. The antioxidant activities of tea extracts were evaluated in terms of H-ORAC (hydrophilic-oxygen radical absorbance capacity) and DPPH (1,1-diphenyl 2-picryl hydrazyl) radical scavenging activity (DPPH-RSA) as well as total polyphenol content and catechins.

\section{Materials and methods}

\section{Plant material}

Green tea (GT) and black tea (BT) samples from eight cultivars were procured from the United Planters' Association of South India (UPASI), Valparai, India, details of which are given in Table 1. These tea cultivars were manufactured to green and black tea by well-defined procedures.

\section{Chemicals}

Reference standard Trolox ${ }^{\circledR}$ (6-hydroxy-2, 5, 7, 8-tetramethylchroman-2-carboxylic acid) and DPPH were purchased from Sigma Chemicals Co., (MO, USA). Chemicals of high grade and purity such as Folin-Ciocalteu reagent (MP Biomedicals; OH, USA), 2-morpho- linoethanesulfonic acid, monohydrate (MES) (Dojindo; Kumamoto, Japan), potassium dihydrogen phosphate, dipotassium hydrogen phosphate, 2, 2'-azobis (2-amidinopropane) dihydrochloride (AAPH) (Sigma) were used in the experiments. Solvents such as hexane, dichloromethane, methanol and acetic acid were procured from Wako Pure Chemical Industries (Osaka, Japan). The standard chemicals of catechins and theaflavins for HPLC analysis were purchased from Mitsui Norin Co., Ltd. (Shizuoka, Japan) and Nagara Science Co., Ltd. (Gifu, Japan), respectively.

\section{Extraction of tea samples with solvents}

Green and black tea samples were pulverized to a coarse powder ( $>300 \mathrm{mesh}$ ) using a knife mill (Millcer800DG, Iwatani Corporation; Tokyo, Japan). The pulverized tea powders $(1 \mathrm{~g})$ were then mixed with sea sand (5 g) and extracted in an automatic solvent extractor (ASE200, Dionex; CA, USA) with hexane-dichloromethane (1:1), and acetic acid acidified aqueous methanol (MWA) where the ratio of methanol, water and acetic acid was maintained as 90:9.5:0.5, sequentially. The MWA extracts were made up to $25 \mathrm{ml}$ and kept at $4^{\circ} \mathrm{C}$ until use.

\section{Polyphenols contents}

The total polyphenols of tea extracts were measured by the modified Folin-Ciocalteu method ${ }^{19}$ using a 96 -well microplate (\#MS-8496F; Sumitomo Bakelite Co., Ltd.; Tokyo, Japan). MWA extracts $(10 \mu \mathrm{l})$ were diluted with $\mathrm{H}_{2} \mathrm{O}(60 \mu \mathrm{l})$ and Folin-Ciocalteu reagent $(15 \mu \mathrm{l})$ was add-

Table 1. Sample tea cultivars and contents of catechins and theaflavins of tea extracts ${ }^{1}$

\begin{tabular}{|c|c|c|c|c|c|c|c|c|c|c|c|c|c|c|c|}
\hline \multirow{3}{*}{$\begin{array}{c}\text { Sample } \\
\text { No. }\end{array}$} & \multirow{3}{*}{$\begin{array}{c}\text { Sample } \\
\text { name }\end{array}$} & \multicolumn{5}{|c|}{ Green tea } & \multicolumn{9}{|c|}{ Black tea } \\
\hline & & \multicolumn{5}{|c|}{ Catechins (mg/g sample) } & \multicolumn{5}{|c|}{ Catechins (mg/g sample) } & \multicolumn{4}{|c|}{ Theaflavins (mg/g sample) } \\
\hline & & EGC & $\mathrm{C}$ & $\mathrm{EC}$ & EGCG & ECG & EGC & $\mathrm{C}$ & $\mathrm{EC}$ & EGCG & ECG & $\mathrm{TF}$ & TF3G & TF3'G & TF3,3'DG \\
\hline 1 & UPASI-2 & 48.8 & $\mathrm{ND}^{2}$ & 11.2 & 78.3 & 19.3 & 16.0 & ND & 3.1 & 7.1 & 3.8 & 12.7 & 15.4 & 4.3 & 7.9 \\
\hline 2 & UPASI-3 & 44.8 & ND & 10.7 & 61.3 & 16.5 & 11.9 & ND & ND & 1.8 & 1.7 & 13.1 & 12.1 & 4.8 & 7.3 \\
\hline 3 & UPASI-6 & 29.9 & ND & 8.9 & 59.6 & 15.7 & ND & ND & ND & 2.7 & 1.6 & 8.0 & 7.9 & 2.4 & 4.9 \\
\hline 4 & UPASI-8 & 38.1 & ND & 10.2 & 78.2 & 21.6 & 20.3 & ND & ND & 6.3 & 3.5 & 13.1 & 16.2 & 5.0 & 11.4 \\
\hline 5 & UPASI-9 & 37.0 & ND & 8.6 & 98.5 & 24.4 & 16.7 & ND & ND & 4.5 & 2.3 & 10.5 & 14.9 & 5.2 & 14.8 \\
\hline 6 & UPASI-10 & 36.2 & ND & 12.5 & 45.0 & 16.0 & 10.9 & ND & ND & 0.9 & 1.1 & 11.1 & 4.6 & 2.2 & 2.9 \\
\hline 7 & TRI-2024 & 33.6 & 1.9 & 11.2 & 90.8 & 33.9 & 13.6 & ND & ND & 5.4 & 9.5 & 9.2 & 10.1 & 2.9 & 9.0 \\
\hline 8 & $\begin{array}{c}\text { Assam } \\
\text { Seedlings }\end{array}$ & 43.5 & 2.1 & 10.2 & 69.1 & 18.3 & 16.1 & ND & ND & 4.9 & 3.0 & 10.4 & 10.2 & 3.7 & 6.9 \\
\hline
\end{tabular}

\footnotetext{
${ }^{1}$ Data are mean values of the duplicate analysis

${ }^{2} \mathrm{ND}$ : not detected
} 
ed to each well. The plate was allowed to stand for $5 \mathrm{~min}$ utes at room temperature. The solution in wells was mixed with $75 \mu \mathrm{l}$ of $2 \%$ (w/v) sodium bicarbonate, kept at room temperature for 15 minutes and the absorbance at $750 \mathrm{~nm}$ was measured by a microplate reader (Powerscan HT, DS Pharma Biomedical; Osaka, Japan). The polyphenol contents was calculated as the gallic acid equivalent (mg GAE / g).

\section{DPPH-radical scavenging activity (DPPH-RSA)}

The DPPH radical scavenging activity of the tea extracts was evaluated according to the procedure described by Blois ${ }^{1}$ with slight modifications by $\mathrm{Oki}^{10}$. Briefly, tea extracts $(100 \mu \mathrm{l})$ or the Trolox ${ }^{\circledR}$ standard (100 $\mu \mathrm{l})$ at different concentrations $(40,80,120$ and $160 \mu \mathrm{M})$ were taken in a 96-well microplate (\#MS-8496F; Sumitomo Bakelite Co., Ltd.). An MES buffer (200 mM, pH $6.0,50 \mu \mathrm{l})$ and $800 \mu \mathrm{M}$ DPPH solution in ethanol $(50 \mu \mathrm{l})$ were added to each well. The plate was allowed to stand at room temperature for 20 minutes and the absorbance was measured at $520 \mathrm{~nm}$ by a microplate reader (Powerscan HT). DPPH radical scavenging activity was expressed as $\mu \mathrm{mol}$ Trolox ${ }^{\circledR}$ equivalent (TE) $/ \mathrm{g}$.

\section{Oxygen radical absorbance capacity (ORAC)}

The ORAC values of the tea extracts were evaluated according to the method of Huangand and others ${ }^{7}$ slightly modified by $\mathrm{Oki}^{11}$.

Trolox ${ }^{\circledR}$ standard and sample solutions of each (20 $\mu \mathrm{l})$ were mixed with a $75 \mathrm{mM}$ potassium phosphate buffer ( $\mathrm{pH} 7.0,20 \mu \mathrm{l})$. Fluorescein solution (94.4 nM, 200 $\mu \mathrm{l})$ was added to each well and the fluorescent intensity (Em: $490 \mathrm{~nm}$, Ex: $535 \mathrm{~nm})\left(f_{0 \mathrm{~min}}\right)$ was measured by a microplate reader (Wallac 1420 ARVOsx, Perkin Elmer; MA, USA). AAPH solution $(31.7 \mathrm{mM}, 75 \mu \mathrm{l})$ was added and the fluorescent intensity was measured every two minutes till $90 \mathrm{~min}$. $\left(f_{2 \text { min. }} \sim f_{90 \text { min }}\right)$. The area under the curve (AUC) was calculated by the formula.

$\mathrm{AUC}=\left(0.5 \times f_{8 \text { min. }}+f_{10 \text { min. }}+f_{12 \text { min. }}+\ldots \ldots .+f_{88 \text { min. }}+\right.$ $\left.0.5 \times f_{90 \text { min }}\right) / f_{0 \text { min. }} \times 2$

Relative ORAC value $(\mu \mathrm{mol} \mathrm{TE} / \mathrm{g})=\left[\left(\mathrm{AUC}_{\text {sample }}-\right.\right.$ $\left.\left.\mathrm{AUC}_{\text {blank }}\right) /\left(\mathrm{AUC}_{\text {Trolox }}-\mathrm{AUC}_{\text {blank }}\right)\right]$ (molarity of Trolox/molarity or weight of sample)

The experimental data on duplicate analyses of average value is reported.

\section{Catechins and theaflavins}

Catechins and theaflavins in the MWA extracts of the tea samples were quantified by HPLC under the following conditions: The column used was a YMC pack Pro ODS ( $5 \mu \mathrm{m}, \phi 4.5 \times 250 \mathrm{~mm}$, YMC; Kyoto, Japan) at $40{ }^{\circ} \mathrm{C}$. The mobile phase [A: $4 \%$ acetonitrile containing
$0.1 \%$ trifluoroacetic acid and B: $60 \%$ acetonitrile containing $0.1 \%$ trifluoroacetic acid, 0 - $30 \mathrm{~min}$. B: 0 - 38.4 \%, 30 - 60 min. B: 38.4 - 60\%, 60 - $88 \mathrm{~min}$. B: $60 \%$ ] at a flow rate of $1.0 \mathrm{ml} / \mathrm{min}$. using the Shimadzu 20A HPLC system. Peaks were observed at a wavelength of $275 \mathrm{~nm}$ using a photo diode array detector and identified from the retention time and UV-spectra compared with standards of catechins and theaflavins.

\section{Contribution of catechins and theaflavins for antioxidant activity}

The contribution of catechins and theaflavins for $\mathrm{H}$ ORAC or DPPH-RSA was calculated by the following formula:

Contribution $(\%)=$ AOA $($ extract $) \div($ AOA $($ component) $\times$ content $) \times 100$

where AOA (extract, $\mu \mathrm{mol} \mathrm{TE} / \mathrm{g}$ sample) is the antioxidant activity of the tea extract, AOA (component, $\mu \mathrm{mol} \mathrm{TE} / \mathrm{mg}$ ) is the antioxidant activity of each catechin or theaflavin, and content ( $\mathrm{mg} / \mathrm{g}$ sample) is the content of each catechin or theaflavin.

\section{Statistical analysis}

The means, standard deviations and Tukey's multiple range tests were calculated using Excel Statistics (Excel-Toukei, Social Survey Research Information Co., Ltd.; Tokyo, Japan) which was Microsoft Excel add-in statistical calculation software.

\section{Results and discussion}

Antioxidant activities and the total polyphenol contents of green and black teas of eight tea cultivars from the UPASI research foundation in India were compared (Fig. 1). The harvested tea leaves were converted to green and black teas via the ordinary manufacturing procedure.

The total polyphenol contents of each sample were measured using the Folin-Ciocalteu method. Among green tea samples, total polyphenol contents ranged from 98.1 (UPASI-3) to 154.6 (TRI-2024) mg GAE/g. Conversely, the total polyphenol contents of black tea ranged from 74.6 (UPASI-6) to 119.4 (UPASI-8) mg GAE/g and were relatively lower than those of green tea. There were no significant differences within 8 cultivars of GT and BT, respectively. A high correlation coefficient $(r=0.913)$ exists for total polyphenol contents in the same cultivar of GT and BT. The manufacturing process for green tea involves exposing fresh leaves to higher temperatures to deactivate the enzymes e.g. polyphenol oxidase. Conversely, the tea leaves were oxidized in the process of making black tea by fermentation. Differences in the 
manufacturing procedures may explain differences in the total polyphenol contents of GT and BT.

It was observed that DPPH-RSA ranged from 1098 to $1376 \mu \mathrm{mol} \mathrm{TE} / \mathrm{g}$ in GT and 508 to $798 \mu \mathrm{mol} \mathrm{TE} / \mathrm{g}$ in BT. UPSAI-2, 8, TRI-2024 and Assam Seedlings showed higher DPPH-RSA for both GT and BT. Conversely, UPASI- 6 and 10 were low. DPPH-RSA showed a very similar trend to the results of total polyphenols contents. The correlation coefficient of total polyphenols and the DPPH-RSA for green and black teas were found to be 0.744 and 0.976 , respectively, suggesting the contribution of polyphenols in BT to DPPH-RSA is more potent than in GT.

Results indicated that H-ORAC values ranged from 2730 to $5031 \mu \mathrm{mol} \mathrm{TE} / \mathrm{g}$ in GT and 1429 to $2766 \mu \mathrm{mol}$ TE/g in BT. Similarly to DPPH-RSA, UPASI-2 showed the highest H-ORAC value and UPASI- 6 and 10 were lowest among the eight tested cultivars. In the ORAC assay, peroxyl radicals (ROO) from AAPH were used to initiate the radical reaction. It is widely recognized that peroxyl radicals in the human body mainly contribute to biological oxidative stress, so the ORAC method is thought to reflect antioxidant reactions in the body, and is recommended by USDA for comparing the antioxidant activities of agricultural products ${ }^{20}$. Comparing the same cultivars, higher antioxidant activities were observed in green rather than black tea samples for both methods i.e. H-ORAC and DPPH-RSA. Correlation coefficients between the parameters of antioxidant activities and polyphenols were provided (Fig. 2); TP/H-ORAC (GT: $r=0.647$, BT: $r=0.790)$, TP/DPPH-RSA $(r=0.744, r=0.976)$ and DPPH-RSA/H-ORAC (GT: $r=0.686, \mathrm{BT}: r=0.811$ ).
1) Total polyphenol

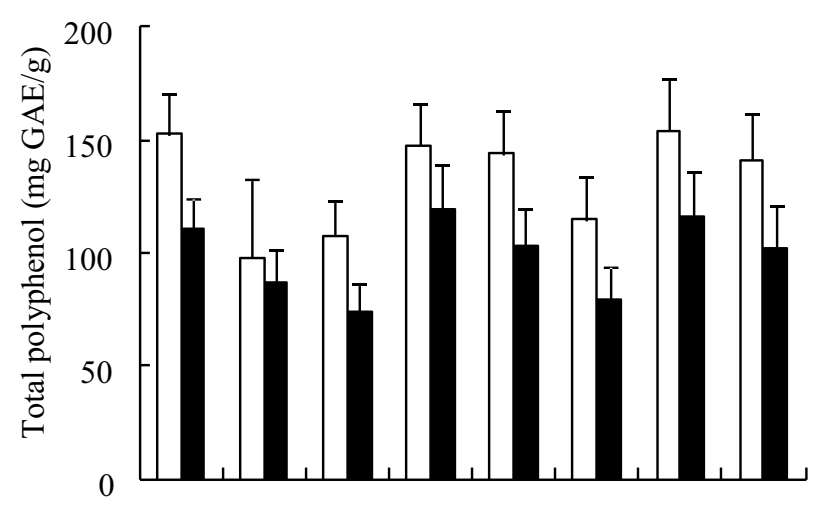

No.1 No. 2 No.3 No.4 No.5 No.6 No.7 No.8

\section{2) DPPH-RSA}

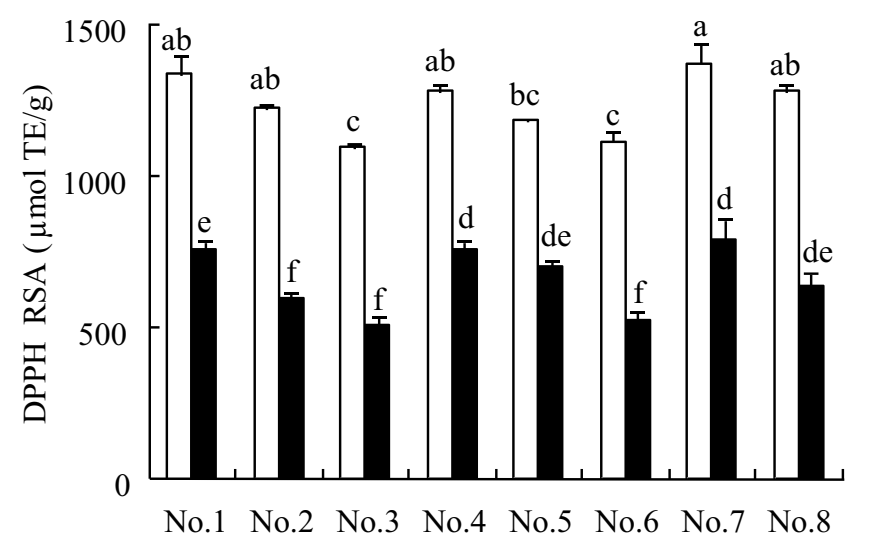

3) H-ORAC value

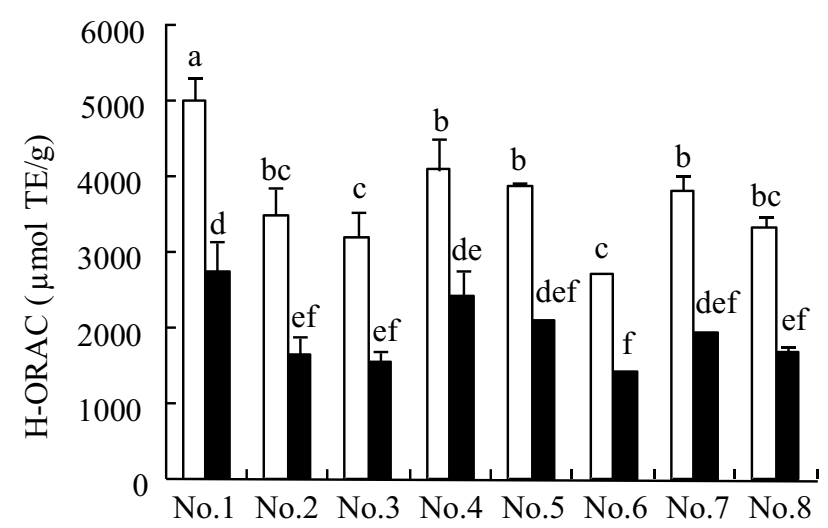

Fig. 1. Total polyphenol content and antioxidant activities in green and black teas

Total polyphenol content 1), DPPH-RSA 2) and H-ORAC values 3) were shown in open (green tea) and closed (black tea) bar. The no. of samples was the same as Table 1. Total polyphenols were measured by the modified Folin-Ciocalteu method and displayed in mg as the gallic acid equivalent. DPPH-RSA and H-ORAC values were displayed by $\mu$ mol of Trolox equivalent. Data were mean and SD. No significant difference between the same characteristics (green and black tea, respectively) was revealed by Tukey's multiple range tests.

$\square$ : Green tea, $\square$ : Black tea 
Therefore, the higher antioxidant activity of GT is partially explained by the increased total polyphenol and catechins contents in GT compared to those of BT. Among the cultivars, higher antioxidant activities were observed in UAPSI-2, TRI-2024, Assam seedlings, UPASI- 8 and 3 in terms of RSA and ORAC.

The contents of five major catechins $((+)$ catechin (C), (-)-epicatechin (EC), (-)-epicatechin gallate (ECG), (-)-epigallocatechin (EGC), (-)-epigallocatechin 3-O-gallate $(\mathrm{EGCG})$ ) and four major theaflavins (only black tea: theaflavin (TF), theaflavin 3-O-gallate (TF3G), theaflavin 3'- $O$-gallate (TF3' $\mathrm{G})$, theaflavin 3,3'-di- $O$-gallate (TF3,3'DG)) were determined by HPLC analysis (Table 1). Contributions of catechins and theaflavins were calculated from the respective quantities and antioxidant activities (DPPH-RSA and H-ORAC of each compound were not shown) of these compounds. Antioxidant activities (DPPH-RSA and H-ORAC) of green tea were mainly derived from ECG and EGCG (Figs. 3 and 4). Conversely, theaflavins, which are formed from catechins during fermentation, showed lower antioxidant activities compared with catechins ${ }^{6}$. In black tea, since the concentration of catechins is low, the major contribution to antioxidant activity came from theaflavins. An abundance of polyphenols with higher antioxidant activity ${ }^{15}$, particularly flavan-3-ols in green and black tea, contribute significantly to its health properties ${ }^{8,23}$. Isolation of these antioxidant compounds from tea might be used for food storage. Tea is also a major source of polyphenols for dietary supplements ${ }^{12}$. Therefore with its antioxidant activity in mind, these results reveal useful information for breeding new tea cultivars.
1) Total polyphenol and H-ORAC

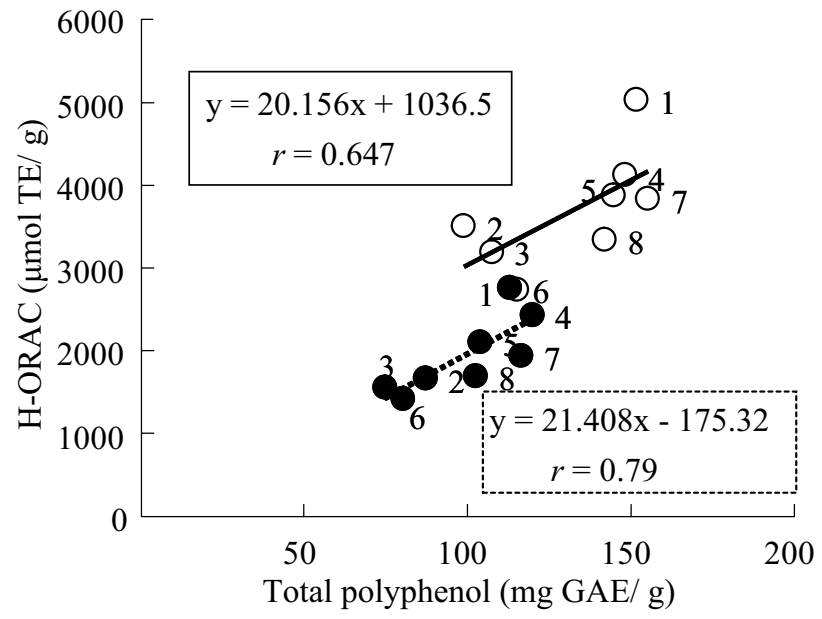

2) Total polyphenol and DPPH-RSA

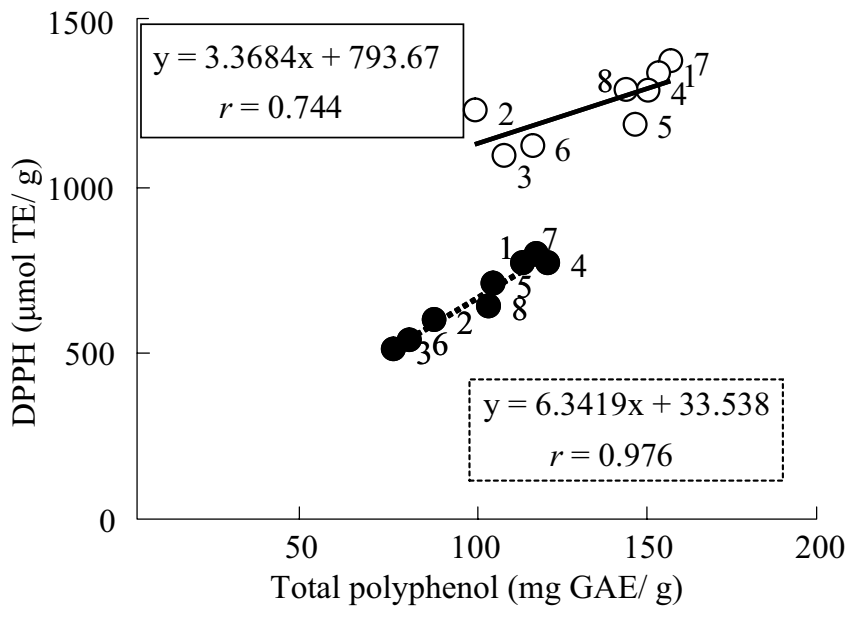

3) DPPH-RSA and H-ORAC value

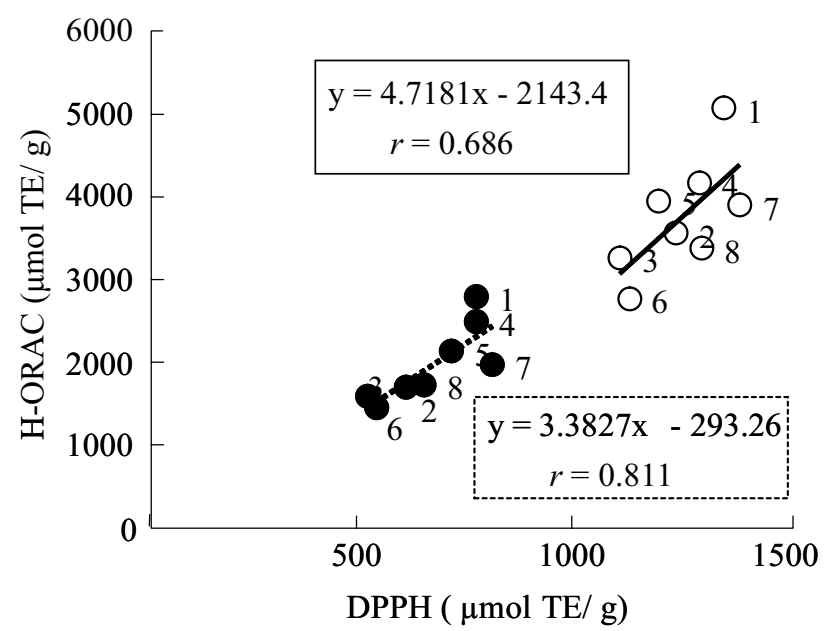

Fig. 2. Correlation between total polyphenol content, H-ORAC value and DPPH-RSA

Green and black teas were plotted by open (green tea) and closed (black tea) circles. The no. of samples was the same as Table 1. Linear regression and correlation of the coefficient were displayed as a line (green tea) and broken line (black tea), respectively. 

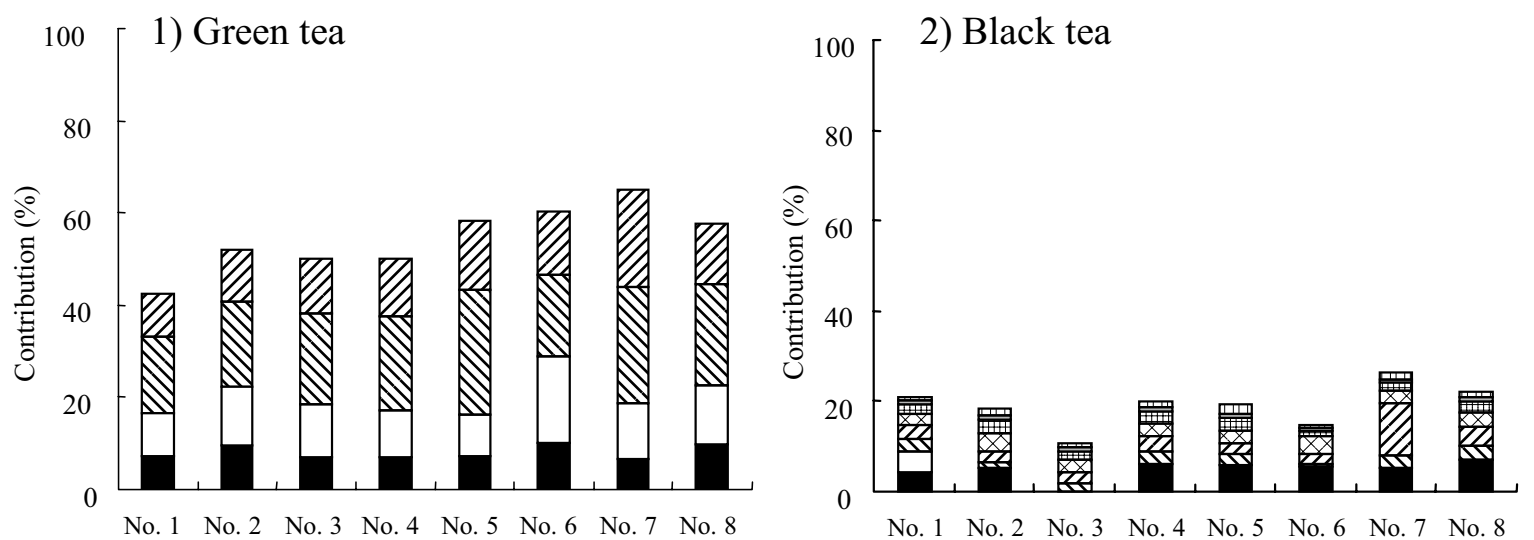

Fig. 3. Contribution of catechins and theaflavins for the H-ORAC value

The contributions of catechins and theaflavins for the H-ORAC of green teal) and black tea2) were calculated based on their amounts, which were measured based on the HPLC and H-ORAC values of each compound. Q7A: ECG, MV: EGCG, $\square$ : EC, $\square$ : EGC, $\square$ : TF-3,3'dg, $\square$ : TF-3'g, $\square$ : TF-3g, $\square$ : TF.
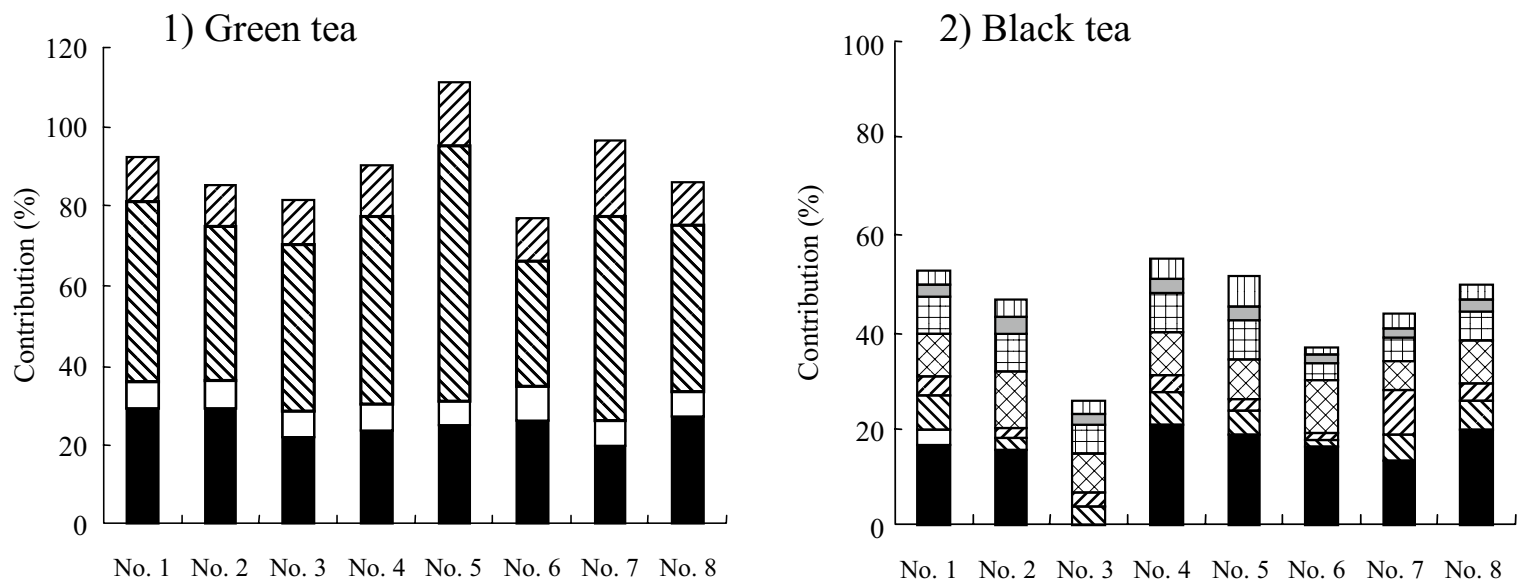

Fig. 4. Contribution of catechins and theaflavins for DPPH-RSA

The contribution of catechins and theaflavins for DPPH-RSA of green tea1) and black tea2) were calculated based on their amounts, which were measured based on the HPLC and DPPH-RSA values of each compound.

Q7A: ECG, MV: EGCG, $\square$ : EC, $\square$ : EGC, $\square$ : TF-3,3'dg, $\square$ : TF-3'g, $\square$ : TF-3g, $\square$ : TF.

\section{Conclusion}

Tea is one of the world's most widely consumed beverages. It is generally consumed as a health-promoting beverage and its health benefits are mainly due to the presence of polyphenols such as catechins in green tea and theaflavins and thearubigins in black tea. Extracts from eight different cultivars of green tea (GT) and black tea (BT) were evaluated for their antioxidant potential using in vitro methods e.g. radical scavenging activity (DPPH-RSA) and hydrophilic oxygen radical absorbance capacity (H-ORAC) as well as being analyzed for total polyphenol (TP) content and catechins. Comparing the same cultivar, GT [H-ORAC: $2730-5031 \mu \mathrm{mol} \mathrm{TE} / \mathrm{g}$; and DPPH-RSA: 1098-1376 $\mu \mathrm{mol} \mathrm{TE} / \mathrm{g}$ ] showed higher antioxidant potential than those of BT (H-ORAC: 1429-2766 $\mu \mathrm{mol} \mathrm{TE} / \mathrm{g}$; and DPPH-RSA: 508-798 $\mu \mathrm{mol} \mathrm{TE} / \mathrm{g}$ ) in in vitro systems, which is partially explained by the increased TP in GT (98.1-154.6 mg GAE/g) compared to BT (74.6-119.4 mg GAE/g) and concentration of catechins. Antioxidant activities were mainly due to catechins in GT and theaflavins in the case of BT. Extracts 
from all cultivars showed good antioxidant potential. Among the cultivars, higher antioxidant activities were observed in UAPSI-2, TRI-2024, Assam seedlings, UPASI- 8 and 3 in terms of RSA and ORAC. However, these need to be confirmed via in vivo systems.

\section{Acknowledgments}

The authors wish to thank the UNITED PLANTERS' ASSOCIATION OF SOUTHERN INDIA for providing the green and black tea samples. The authors also greatly thank Ms. M. Yamamoto and Ms. Y. Kojima for their excellent technical assistance. This work was supported by a grant-in-aid for the 'Development of evaluation and management methods for the supply of safe, reliable and functional food and firm produce' from the Ministry of Agriculture, Forestry and Fisheries of Japan.

\section{References}

1. Blois, M.S. (1958) Antioxidant determinations by the use of a stable free radical. Nature, 181, 1199-1200.

2. Denham, H. (1956) Aging: A theory based on free radical and radiation chemistry. J. Gerontol. 11, 298-300.

3. Emilio, R. (2009) Nuts and novel biomarkers of cardiovascular disease. Amer. J. Clin. Nutr., 89, 1649S-1656S.

4. Hancock, J., Desikan, R., Neil, S. (2001) Role of reactive oxygen species in cell signaling pathways. Biochem. Biomed. Asp. of Oxidase Modif., 29, 345-350.

5. Hara, Y. et al. (1995) VI, Biochemistry of processing black tea. Food Rev. Int., 11, 457-471.

6. Hashimoto, F. et al. (2003) Evaluation of the antioxidative effect (in vitro) of tea polyphenols. Biosci. Biotechno. Biochem., 67, 396-401.

7. Huang, D. et al. (2002) High-throughput assay of oxygen radical absorbance capacity (ORAC) using a multichannel liquid handling system coupled with a microplate fluorescence reader in 96-well format. J. Agric. Food Chem., 50, 4437-4444.

8. Kurihara, H. et al. (2003) Inhibitory effect of oolong tea on the oxidative state of low density lipoprotein (LDL). Biol. Pharma Bull., 26, 739-742.

9. Mora, A. et al. (1990) Structure-activity relationships of polymethoxyflavones and other flavonoids as inhibitors of non-enzymic lipid peroxidation. Biochem. Pharmacol., 40, 793-797.
10. Oki, T. (2008) Evaluation method of DPPH radical scavenging activity. Man. Eval. Method of Food Funct., II, 7178.

11. Oki, T. (2008) Evaluation method of ORAC. Man. Eval. Method of Food Funct., II, 79-86.

12. Rechner, A.R. et al. (2002) Black tea represents a major source of dietary phenolics among regular tea drinkers. Free Radic. Res., 36, 1127-1135.

13. Roy, M. et al. (2010) ORAC and DPPH assay comparison to assessantioxidant capacity of tea infusions: Relationship between total polyphenol and individual catechin content. Int. J. Food Sci. Nutr., 61, 109-124.

14. Seeram, N. et al. (2008) Comparison of antioxidant potency of commonly consumed polyphenol-rich beverages in the United States. J. Agric. Food Chem., 56, 1415-1422.

15. Seeram, N.P. et al. (2006) Catechin and caffeine content of green tea dietary supplements and correlation with antioxidant capacity. J. Agric. Food Chem., 54, 1599-1603

16. Sen, C.K. \& Packer, L. (1996) Antioxidant and redox regulation of gene transcription. J. Fed. Am. Soc. Exp. Biol., 10, 709-720.

17. Subramanian, N. et al. (1999) Role of polyphenol oxidase and peroxidase in the generation of black tea theaflavins. $J$. Agric. Food Chem., 47, 2571-2578.

18. Takabe, W. et al. (2001) Oxidative stress promotes the development of transformation: Involvement of a potent mutagenic lipid peroxidation product, acrolein. Carcinogenesis, 22, 935-941.

19. Tsuruta, H. et al. (2007) Studies of development of food materials from unused resource - in vitro evaluations of bioactivities on rotas root. Res. Rep. Ind. Tech. Cent. Saga Prefect., 36-38.

20. USDA Government: United States Department of Agriculture: Agricultural Research Service, Products \& Services, Oxygen Radical Absorbance Capacity (ORAC) of Selected Foods, Release 2. http://www.ars.usda.gov/SP2UserFiles/ Place/12354500/Data/ORAC/ORAC_R2.pdf

21. Varnam, A.H. \& Sutherland, J.P. (1994) Beverages: Thechnology, Chemistry and Microbiology. Chapman and Hall, New York, London, USA, UK, 126-190.

22. Vendeland, J.S. (1996) Tea Horticulture and Black Tea Processing. World Coffee and Tea. 18-22.

23. Wang, Y. \& Ho, C.T. (2009) Polyphenolic chemistry of tea and coffee: a century of progress. J. Agric. Food Chem., 57, 8109-8114.

24. Zabirnyk, O. et al. (2010) Oxidized low-density lipoproteins upregulate proline oxidase to initiate ROS-dependent autophagy. Carcinogenesis, 31, 446-454. 